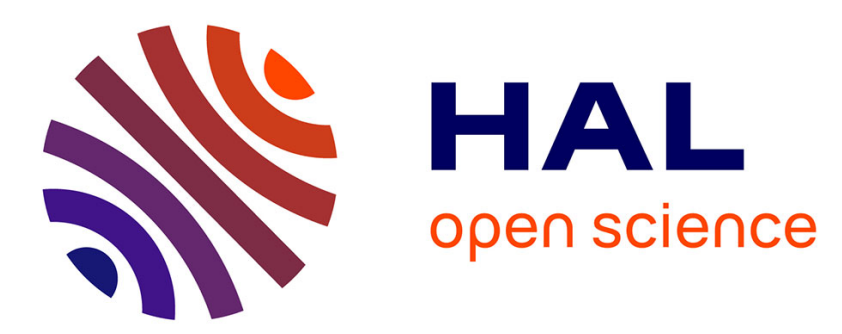

\title{
Belle Aire Sud à Aytré (Charente-Maritime). Une série de céramiques de la phase moyenne du premier âge du Fer
}

Christophe Maitay, Jean-Paul Nibodeau

\section{- To cite this version:}

Christophe Maitay, Jean-Paul Nibodeau. Belle Aire Sud à Aytré (Charente-Maritime). Une série de céramiques de la phase moyenne du premier âge du Fer. Aquitania, 2009, t. 25, p. 7-19. hal-02132782

\section{HAL Id: hal-02132782 \\ https://hal.science/hal-02132782}

Submitted on 28 May 2019

HAL is a multi-disciplinary open access archive for the deposit and dissemination of scientific research documents, whether they are published or not. The documents may come from teaching and research institutions in France or abroad, or from public or private research centers.
L'archive ouverte pluridisciplinaire HAL, est destinée au dépôt et à la diffusion de documents scientifiques de niveau recherche, publiés ou non, émanant des établissements d'enseignement et de recherche français ou étrangers, des laboratoires publics ou privés. 
Christophe Maitay avec la collaboration de Jean-Paul Nibodeau

\section{Belle Aire Sud à Aytré (Charente-Maritime). Une série de céramiques de la phase moyenne du premier âge du Fer}

RÉSUMÉ

À l'occasion d'un diagnostic archéologique effectué sur la commune d'Aytré, près de La Rochelle (Charente-Maritime), a été découverte une fosse creusée dans le calcaire, qui a livré un mobilier céramique homogène accompagné de quelques fragments d'objets en alliage cuivreux associés à une étape de la métallurgie du bronze. L'étude des formes céramiques, notamment un gobelet à panse segmentée, permet de dater cet ensemble homogène de la phase moyenne du premier âge du Fer (Hallstatt D1). Cette période, encore mal connue dans l'ouest de la France, est marquée par l'abandon progressif des traditions céramiques de l'âge du Bronze et par le développement de nouvelles formes de vases. La série de céramiques d'Aytré, bien que modeste, illustre parfaitement cette étape de la Protohistoire régionale. Elle vient par ailleurs documenter un secteur pour lequel nous n'avions jusqu'alors que peu d'informations.

\section{Mots-CLÉS}

Centre-ouest de la France, Charente-Maritime, premier âge du Fer, structure excavée, céramique, typologie, alliage cuivreux, métallurgie.

\section{AbSTRACT}

At an archaeological diagnosis carried out at Aytré, near La Rochelle (Charente-Maritime Department), a pit dug into the limestone revealed homogeneous ceramic furniture with a few fragments of copper alloy. The study of ceramic shapes, including a cup with segmented belly, allows the ensemble to be dated to the middle phase of the First Iron Age (Hallstatt D1). This period, relatively poorly documented in the region, is characterized by the progressive abandonment of Bronze Age ceramic traditions and by the development of new forms of vases. This series of ceramics, although modest, illustrates this phase of the First Iron Age; furthermore it provides documentation for a sector for which we had previously only limited information.

\section{KEYWORDS}

West Central France, Charente-Maritime, First Iron Age, pit, ceramic, pottery typology, copper-alloy, metallurgy. 


\section{INTRODUCTION}

Depuis plusieurs années, l'extension urbaine et industrielle de l'agglomération rochelaise s'accompagne d'opérations de diagnostic et de fouilles préventives réalisées par l'INRAP et par des opérateurs privés ou territoriaux : camp néolithique du Fief des Quatre Chevaliers à Périgny ${ }^{1}$, enclos fossoyés du Bronze ancien de la Vaurie à Périgny ${ }^{2}$, habitat rural du premier âge du Fer de Dompierre-sur-Mer ${ }^{3}$, habitat littoral gaulois des Ormeaux à Angoulins ${ }^{4}$, pour n'en citer que quelques-unes. Dans le cadre de l'aménagement de la ZAC de Belle Aire Sud, à Aytré, à quelques kilomètres du centre-ville de La Rochelle, une vingtaine de structures excavées ont pu être dégagées $^{5}$. L'une de ces fosses a livré un ensemble homogène de poteries attribuées à la phase moyenne du premier âge du Fer.

Si nous disposons d'une documentation plus importante pour la phase finale (Hallstatt D2/D3 - début de La Tène ancienne), les phases ancienne (Hallstatt C) et moyenne du premier âge du Fer (Hallstatt D1) restent encore aujourd'hui peu documentées. La série présentée, modeste mais issue d'un milieu clos, apporte de nouveaux éléments sur le premier âge du Fer dans le centre-ouest de la France ${ }^{6}$.

\section{Cadres de l'opération et CONTEXTES}

La découverte a eu lieu au cours d'un diagnostic archéologique conduit par l'INRAP en début d'année 2008, et faisant suite au projet d'extension de la ZAC de Belle Aire Sud? ${ }^{7}$ Les parcelles concernées sont situées au lieu-dit la Grande Couture, à l'est du bourg d'Aytré, à la périphérie occidentale du plateau aunisien (fig. 1 et 2 ). Le point culminant ne dépasse pas $8 \mathrm{~m} \mathrm{NGF}$ (fig. 2). Le substrat se compose d'une alternance de bancs de calcaire dur se délitant en plaquettes et de bancs marneux plus tendres du Kimméridgien inférieur ${ }^{8}$. En surface, le rocher est très fracturé. Il s'arrache en petits blocs centimétriques ou en plaquettes décimétriques liés par un sédiment argilo-limoneux de couleur brun rouille à brun orangé.

La position topographique du site, un plateau calcaire cerné à l'ouest par les marais d'Aytré, au sud par le marais du Pont de la Pierre, et à l'est par un petit vallon où coule l'Otus, en fait une zone propice à l'implantation humaine. Des vestiges protohistoriques sont représentés par deux sites à sel implantés en bordure du marais ${ }^{9}$ et par un statère gaulois conservé dans une collection privée ${ }^{10}$. Les vestiges d'une villa gallo-romaine ont été reconnus sur plusieurs centaines de mètres carrés, à moins de $150 \mathrm{~m}$ au sud-est du site de Belle Aire Sud.

Le nombre de structures mises au jour pendant cette opération (23) s'avère très modeste au regard de la surface considérée $\left(44553 \mathrm{~m}^{2}\right.$ dont $4590 \mathrm{~m}^{2}$ diagnostiqués). Il s'agit de trous de poteau creusés dans le calcaire, de fosses isolées et peu profondes, de fossés à profil en $\mathrm{V}$ ou en $\mathrm{U}$ et de concentrations de pierres brûlées. Le remplissage des structures en creux a livré quelques tessons de céramiques protohistoriques ${ }^{11}$, antiques et médiévales. Seule l'une des fosses, une excavation située à l'extrémité ouest des terrains sondés (fig. 2), a livré un mobilier céramique significatif du premier âge du Fer. Deux des tranchées du diagnostic ont fourni quant à elles plusieurs gros morceaux d'argile cuite dont certains semblent avoir été lissés (éléments de torchis? parois de fours ?). Des tracés linéaires peuvent être attribués à des aménagements agraires d'époque médiévale ou moderne (fossés parcellaires).

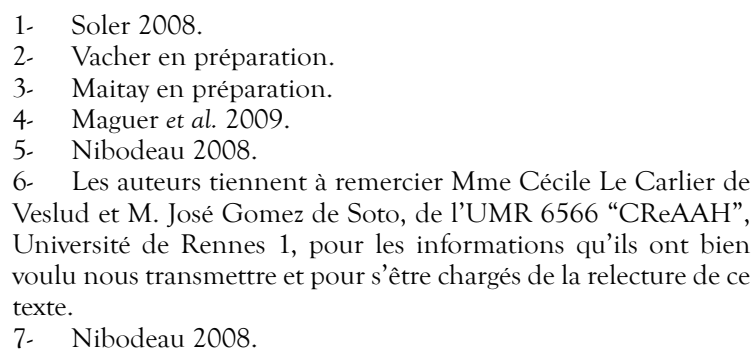
Veslud et M. José Gomez de Soto, de l'UMR 6566 "CReAAH", Université de Rennes 1, pour les informations qu'ils ont bien voulu nous transmettre et pour s'être chargés de la relecture de ce

7. Nibodeau 2008. texte.
8- Hantzpergue 1988.

9. Le premier est localisé à $900 \mathrm{~m}$ au sud-ouest, le second à 300 au sud, non loin de la ferme de la Grenouillière (prospections M. Lavergne, 1993).

10. Maurin 1999, 95

11- Structures 5, 6, 7 et 15 (tranchée 11). Ces tessons de poteries ne présentent pas de forme ou de décor déterminant et n'apportent pas ici d'information complémentaire. 


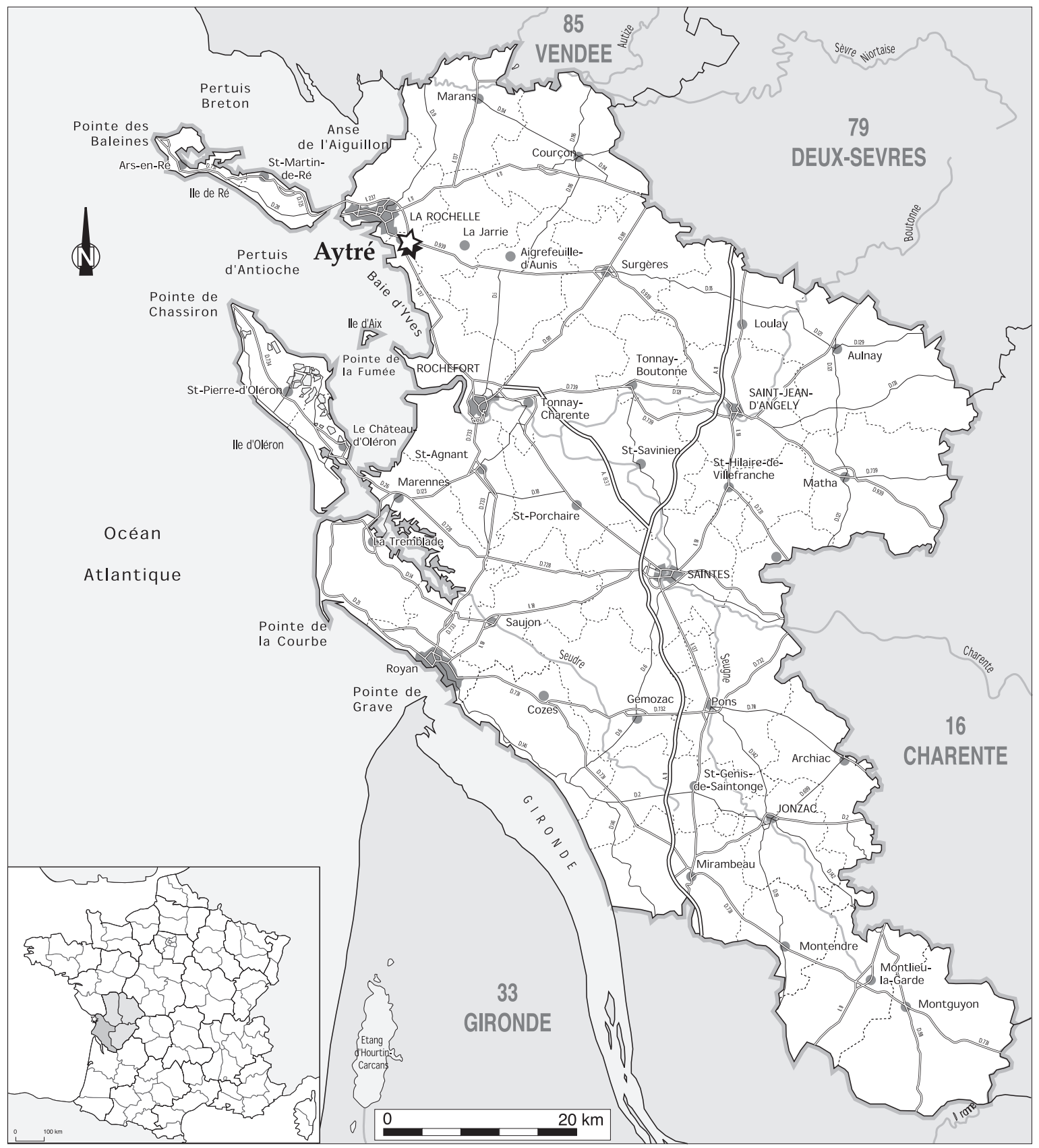

Fig. 1. Localisation du site de Belle Aire Sud à Aytré, Charente-Maritime (DAO M. Coutureau, INRAP). 


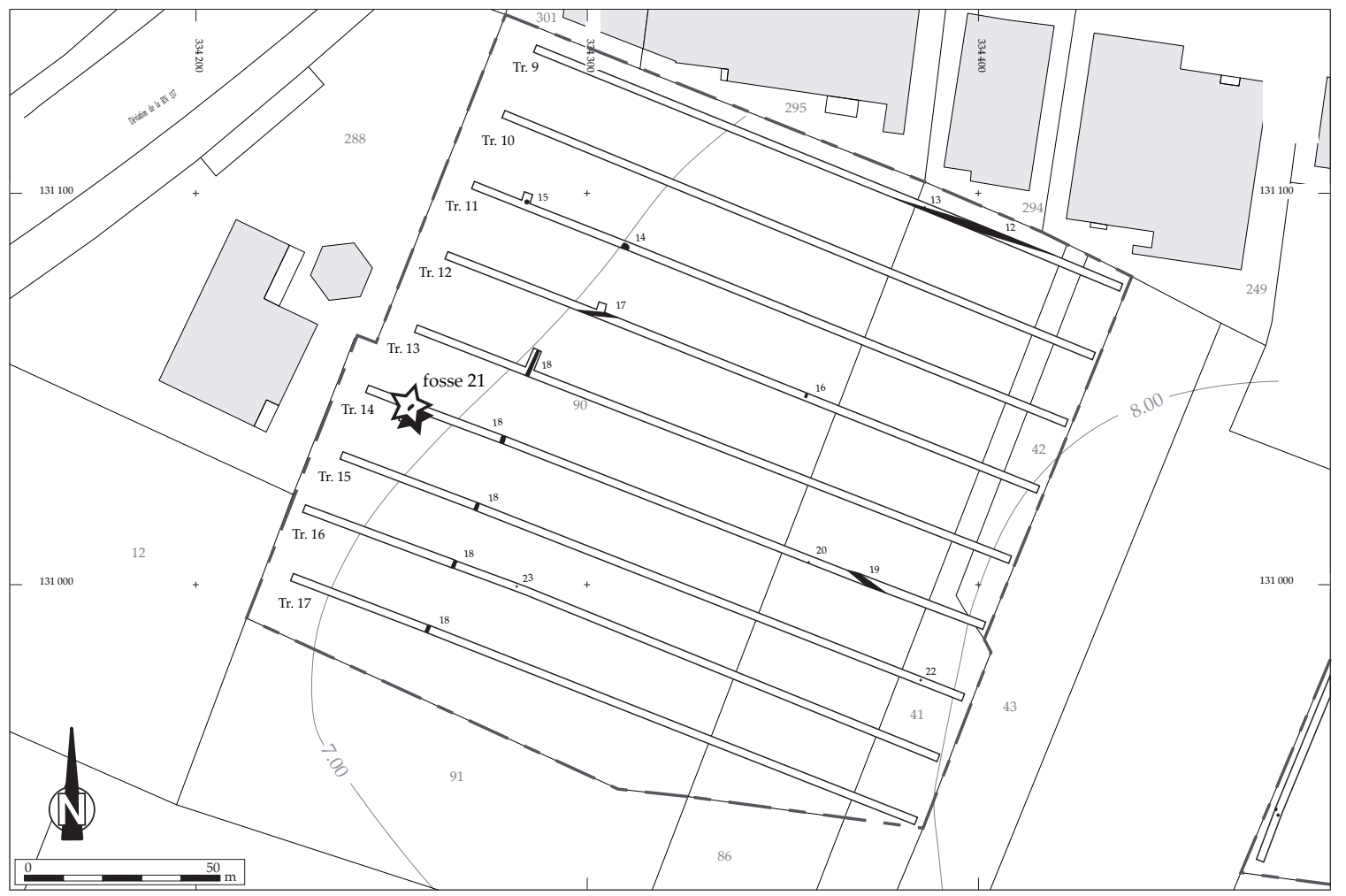

Fig. 2. ZAC de Belle Aire Sud à Aytré, Charente-Maritime. Emplacement de la fosse 21, zone $B$, tranchée 14 (relevé V. Miailhe, DAO M. Coutureau, INRAP).

\section{UNE STRUCTURE DE L'ÂGE DU FER : LA FOSSE F21}

La fosse F21 se situe à l'extrémité occidentale de la tranchée de diagnostic $n^{\circ} 14$. Rien n'atteste sa contemporanéité avec les autres structures fouillées sur le site. Elle était recouverte par un niveau hétérogène de $0,30 \mathrm{~m}$ de terre arable. La structure, creusée dans le substrat calcaire, possède une forme ovale, des rebords très irréguliers et un fond aplati (fig. 3) ; son axe principal est orienté $\mathrm{NO} /$ SE. Elle mesure $0,95 \mathrm{~m}$ de longueur, $0,70 \mathrm{~m}$ de largeur et $0,40 \mathrm{~m}$ de profondeur. Environ $80 \%$ de son comblement a été prélevé et intégralement tamisé à l'eau.

La partie supérieure du remplissage est composée d'un niveau de limon brun contenant quelques blocs de calcaire de petit module (US 01). Son épaisseur n'excède pas 0,08 $\mathrm{m}$. L'US 01 recouvre une couche constituée de sédiment sablo-limoneux noir et de blocs de pierre de moyen module, très riche en cendres et en particules de charbon de bois (US 02), qui occupait environ $90 \%$ du volume de la fosse et recouvrait une couche fine et meuble de limon brun clair n'ayant livré que peu de mobilier archéologique (US 03). L'US 02 contenait quelques morceaux centimétriques d'argile cuite présentant une face lissée plane ou concave pouvant correspondre à des fragments de torchis, de sole de foyer ou de parois de four. Un morceau de fer, quelques scories, ainsi que des gouttelettes et des fragments d'objets en alliage cuivreux proviennent également de ce niveau. Aucune trace de rubéfaction n'a été observée sur les parois de la fosse. 

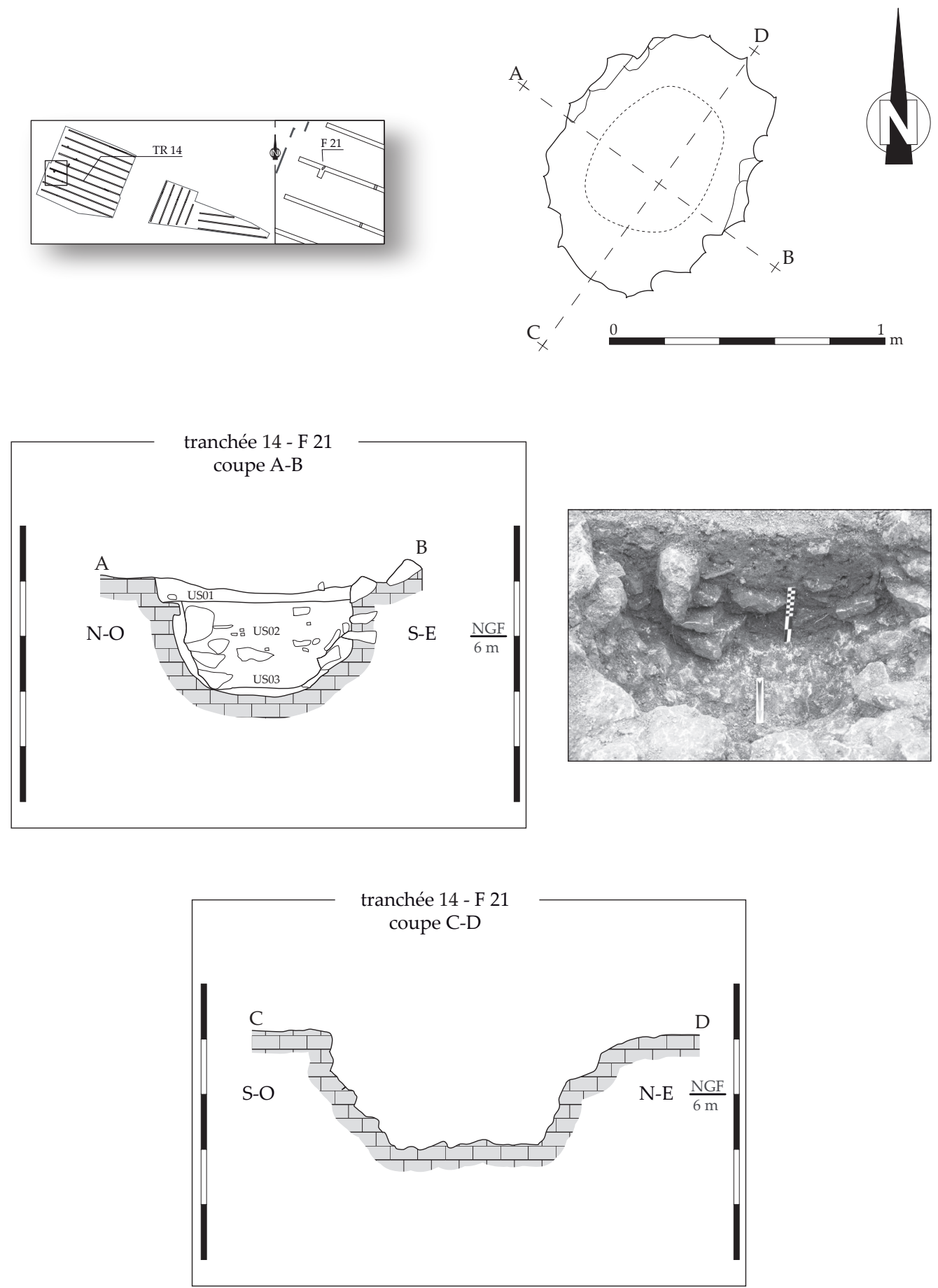

Fig. 3. ZAC de Belle Aire Sud à Aytré, Charente-Maritime. Plan et coupe de la fosse 21 (relevé J.-P. Nibodeau et C. Pelletier, DAO M. Coutureau, INRAP). 


\section{LE MOBILIER DE LA FOSSE 21}

\section{Les vases en terre cuite}

687 tessons correspondant à un NMI de 18 vases ont été inventoriés dans le remplissage de la fosse 21 (fig. 4). Ils représentent un poids de $4259 \mathrm{~g}$ et proviennent pour la plupart de l'US 02. Leur état de conservation est inégal, mais la plupart des fragments présentent des surfaces et des cassures érodées. Aucun moyen de préhension n'a été observé dans cet ensemble. L'état de fragmentation du mobilier et la mauvaise conservation des parois des tessons n'ont pas permis d'effectuer de remarques liées au façonnage de ces vases. Une observation à l'œil nu permet en revanche de déterminer trois principaux groupes de pâtes en fonction de la fréquence et de la taille des inclusions.

Le groupe 1 possède une pâte argileuse à inclusions grossières de nature minérale contenant des paillettes de mica et de nombreux grains hétérométriques, de couleur blanche à gris blanc cassé, réparties uniformément dans la matrice. La présence de nombreux fragments de coquilles fossiles pourrait indiquer, comme pour les groupes 2 et 3, l'emploi d'une argile d'origine sédimentaire. Les surfaces sont simplement lissées et la cuisson, réductrice et bien menée, a conféré aux parois et aux cœurs des tessons une couleur grise à noire.

Les deux autres groupes rassemblent des céramiques possédant une pâte similaire, mais avec des inclusions beaucoup moins fréquentes (groupe 2 : pâte argileuse à texture semi-fine ; groupe 3 : pâte argileuse à texture fine, dégraissant composé principalement de quartz et de petites paillettes de micas). Toutes ces céramiques sont façonnées manuellement.

\section{INVENTAIRE DU MOBILIER :}

\section{$\mathrm{N}^{\circ} 1$ - F21/US 01}

Fragments d'un petit vase à épaulement marqué, col subvertical et lèvre arrondie (fig. $\left.4 a, n^{\circ} 1\right)$. Diamètre de l'ouverture : $11 \mathrm{~cm}$; diamètre maximum de la panse : $13 \mathrm{~cm}$. La carène est ornée d'une série de fines incisions obliques. Surface externe de couleur orangée, interne de couleur gris beige et le cœur de couleur grise (cuisson oxydo-réductrice). Groupe 3.

\section{$\mathrm{N}^{\circ} 2$ - F21/US 02}

Partie supérieure d'un vase à épaulement, col concave et lèvre arrondie (fig. $4 \mathrm{a}, \mathrm{n}^{\circ} 2$ ). Diamètre de l'ouverture : env. $25 \mathrm{~cm}$; diamètre maximum de la panse : env. $27 \mathrm{~cm}$. Surfaces et cœur de couleur gris-noir (post-cuisson réductrice). Comme pour le vase précédent, seules les surfaces externes ont été lissées, l'intérieur n'ayant été que sommairement raclé (traces d'outils encore visibles). Groupe 2.

\section{$\mathrm{N}^{\circ} 3$ - F21/US 02}

Fragment de jatte à panse convexe et lèvre aplatie (fig. 4a, $\mathrm{n}^{\circ} 3$ ). Diamètre de l'ouverture (diamètre maximum) : env. $25 \mathrm{~cm}$. Surfaces et cœur de couleur grise (post-cuisson réductrice). Parois internes et externes lissées. Groupe 2.

\section{$\mathrm{N}^{\circ} 4$ - F21/US 02}

Fragments de col évasé dont la lèvre est aplatie et surépaissie (fig. $4 \mathrm{a}, \mathrm{n}^{\circ} 4$ ). Diamètre de l'ouverture : $34 \mathrm{~cm}$. Les surfaces externes sont de couleur brune, les parois internes et le cœur de couleur grise. Parois internes et externes lissées. Groupe 2.

\section{$\mathrm{N}^{\circ} 5$ - F21/US 02}

Fragment de la partie supérieure d'un grand vase de stockage à col évasé et lèvre biseautée (fig. $4 \mathrm{a}, \mathrm{n}^{\circ}$ 5). Diamètre de l'ouverture : $38 \mathrm{~cm}$. Les jonctions entre les colombins sont perceptibles sur les parois internes et externes du col. Surfaces de couleur gris-noir (post-cuisson réductrice). Groupe 1.

\section{$\mathrm{N}^{\circ} 6-\mathrm{F} 21 / \mathrm{US} 02$}

Fragment de la partie supérieure d'un vase à col évasé et lèvre digitée (fig. $4 \mathrm{~b}, \mathrm{n}^{\circ}$ 6). Diamètre non restituable. Surfaces externes marron, internes noires et cœur gris (post-cuisson réductrice). Groupe 2.

\section{$\mathrm{N}^{\circ} 7$ et $8-\mathrm{F} 21 / \mathrm{US} 02$}

Ces deux tessons appartiennent à deux récipients dont la partie supérieure de la panse, ou bien la jonction entre la panse et le col, est ornée d'un cordon digité (fig. 4b, nº 7 et 8). Céramique à parois épaisses. Couleur externe : brun-marron, interne : gris-beige, cœur : gris. Cuisson réductrice. Groupe 1.

\section{$\mathrm{N}^{\circ} 9$ - F21/US 02}

La partie externe de cette base aplatie est ornée d'une ligne de fines incisions (fig. $4 \mathrm{~b}, \mathrm{n}^{\circ} \mathrm{9}$ ). Diamètre de la base : env. $8 \mathrm{~cm}$. Surfaces externes de couleur brun-gris, surfaces internes et cœur gris (post-cuisson réductrice). Surfaces lissées. Groupe 2. 


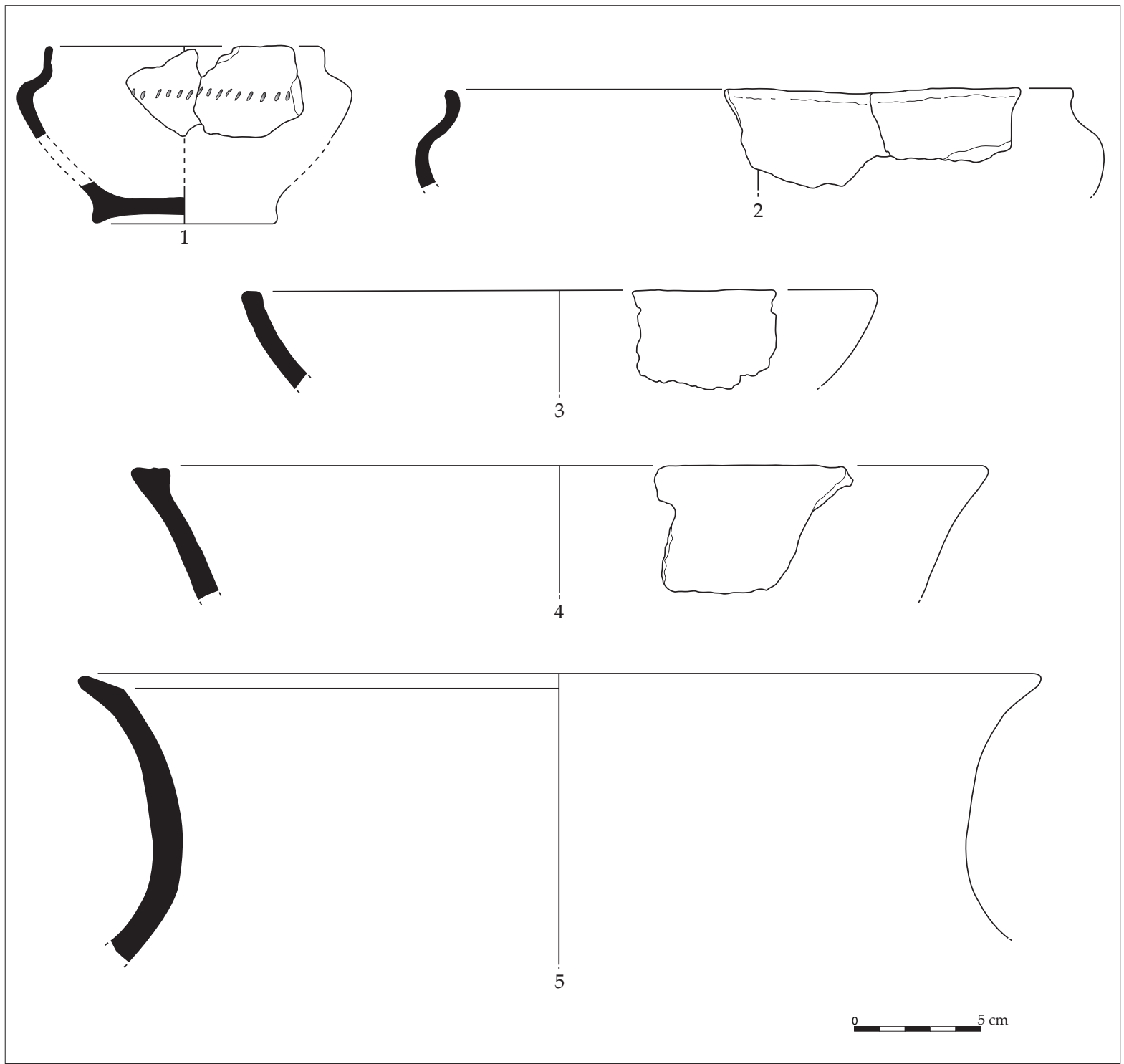

Fig. 4a. ZAC de Belle Aire Sud à Aytré, Charente-Maritime.

Mobilier céramique de la fosse 21 (dessin et DAO Chr. Maitay, INRAP). 


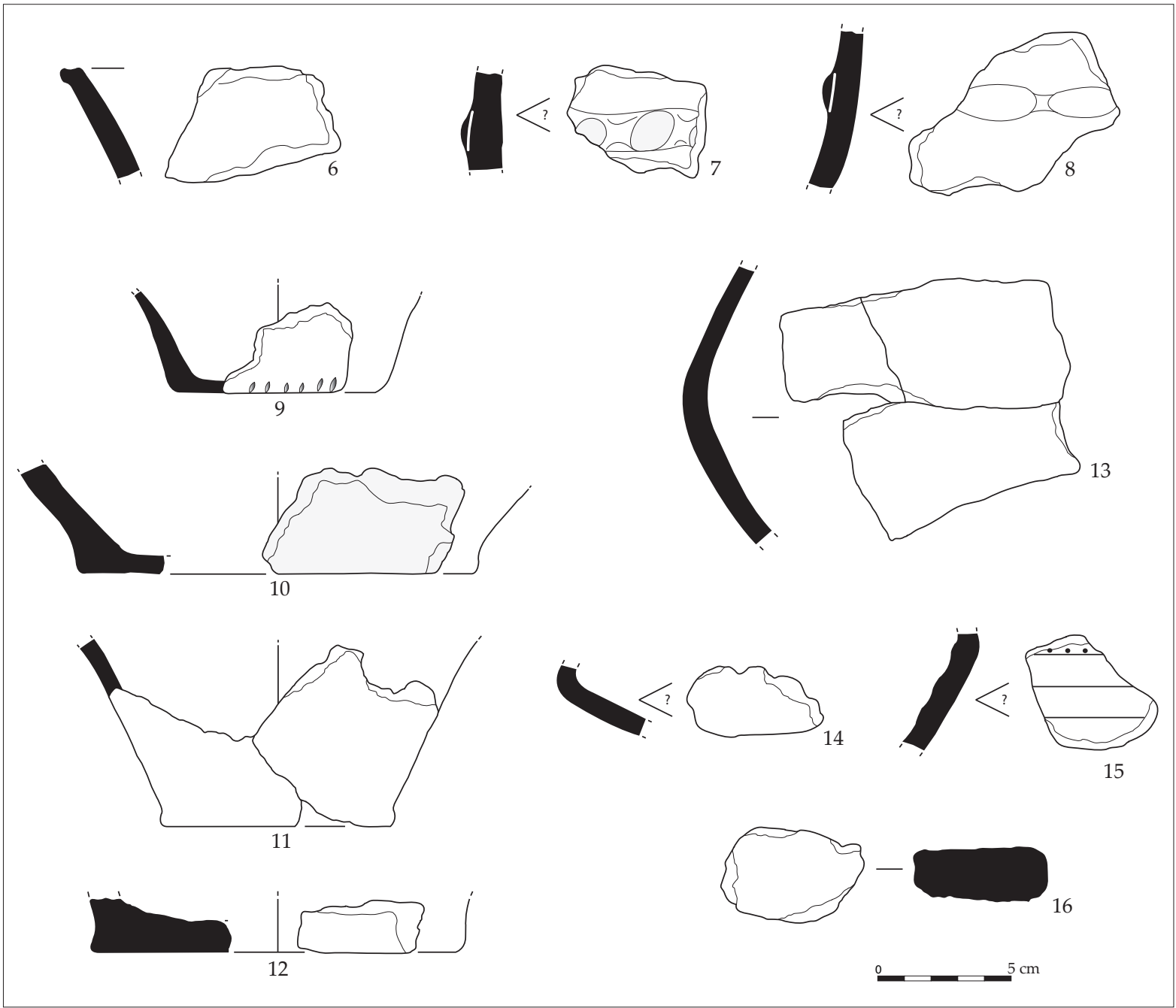

Fig. 4b. ZAC de Belle Aire Sud à Aytré, Charente-Maritime.

Mobilier céramique de la fosse 21 (dessin et DAO Chr. Maitay, INRAP). 


\section{$\mathrm{N}^{\circ} 10-\mathrm{F} 21 / \mathrm{US} 01$}

Partie inférieure d'un vase à fond plat (fig. $4 b, n^{\circ} 10$ ). Diamètre de la base : env. $15 \mathrm{~cm}$. Pâte semi-fine (grains de calcaire et micro-fragments de coquilles) et cuisson réductrice. Surface externe lissée. Groupe 2.

\section{$\mathrm{N}^{\circ} 11-\mathrm{F} 21 / \mathrm{US} 02$}

Partie inférieure d'un vase à fond plat et parois rectilignes (fig. $4 \mathrm{~b}, \mathrm{n}^{\circ} 11$ ). Diamètre de la base : env. $9 \mathrm{~cm}$. La jonction entre le fond et la panse est marquée intérieurement par une cannelure irrégulière. Parois externes lissées. Cour et surfaces de couleur noire mate. Groupe 2.

\section{$\mathrm{N}^{\circ} 12$ - F21/US 03}

Deux fragments de fond plat appartenant à un même vase (fig. $4 \mathrm{~b}, \mathrm{n}^{\circ} 12$ ). Diamètre de la base : env. $14 \mathrm{~cm}$. Couleur externe et interne : gris-orangé, cour : gris. Cuisson oxydo-réductrice. Groupe 1.

\section{$\mathrm{N}^{\circ} 13$ - F21/US 02}

Tessons appartenant à un vase dont la panse possède une angulation douce (fig. $4 \mathrm{~b}, \mathrm{n}^{\circ} 13$ ). Couleur externe brun-gris, interne gris-noir, cœur gris-noir. Post-cuisson réductrice. Surfaces lissées. Groupe 2.

\section{$\mathrm{N}^{\circ} 14-\mathrm{F} 21 / \mathrm{US} 01$}

Un fragment de panse carénée (fig. 4b, n¹4). Traitement de surface très sommaire. Groupe 2.

\section{$\mathrm{N}^{\circ} 15$ - F21/US 02}

Un fragment de partie supérieure de panse ornée de trois cannelures jointives peu profondes (fig. $4 \mathrm{~b}, \mathrm{n}^{\circ} 15$ ). Ce décor est surmonté d'une ligne de petites impressions circulaires prenant place à la jonction du col et de la panse. Surfaces internes et externes lissées. Couleur externe brun-orangé, interne gris-noir, cœur gris-clair. Petites taches noires dans la pâte (matière organique? oxydes de fer ?). Groupe 3.

\section{$\mathrm{N}^{\circ} 16$ - F21/US 02}

Deux fragments de fond plat appartenant à un même vase (non dessiné). Diamètre de la base et départ de la panse non restituable. Surfaces de couleur gris-orangé et cœur gris. Groupe 2.

\section{$\mathrm{N}^{\circ} 17$ et $18-\mathrm{F} 21 / \mathrm{US} 02$}

Deux fragments de bord à lèvre arrondie et aplatie (non dessinés). Groupe 2.

\section{$\mathrm{N}^{\circ} 19$ - F21/US 02}

Fragment d'argile cuite présentant une face supérieure lissée, et une face inférieure irrégulière et rugueuse (fig. 4b, $\mathrm{n}^{\circ} 16$ ). Dimensions : 5,1 x 3,5 × $2 \mathrm{~cm}$. Couleur brun clair et post-cuisson oxydante. Probable élément de sole de foyer.

\section{Le mobilier métallique}

Le comblement de la fosse 21 contenait également de petits fragments de bronze de plusieurs millimètres à quelques centimètres de longueur maximale. L'US 01 a livré trois fragments de tiges plates et des déchets de fabrication (fig. 5, n³), l'US 02 des fragments de fines plaques en alliage cuivreux (fig. 5 , $\mathrm{n}^{\circ} 1$ et 2 : éléments de placage, déchets de fabrication?) et de tiges de section quadrangulaire ou circulaire (fig. $5, n^{\circ} 4$ à 7 ), et l'US 03 un fragment de tige de section carrée et des déchets de fonte de petite taille (fig. $5, n^{\circ} 8$ à 10 ). Le tamisage des sédiments a permis d'enrichir cet inventaire d'une cinquantaine de petites gouttes de métal et de plusieurs petites scories de bronze. Le poids total de métal n'excède pas 50,63 g (US $01: 7,22 \mathrm{~g}$; US $02: 36,88 \mathrm{~g}$; US $03: 6,53 \mathrm{~g}$ ).

Des petits morceaux de métal amalgamés à de l'argile de couleur claire montrent des traces évidentes d'altération thermique (texture aérée et aspect scorifié, poids très faible). Ils pourraient appartenir à des creusets en terre cuite fortement vitrifiés, ou bien à des fragments d'extrémités distales de tuyères. Les nombreuses gouttes en alliage cuivreux résultent de la coulée du métal en fusion. Contrairement aux cônes de coulée, elles ne sont que rarement récupérées et se retrouvent couramment dans le sol des ateliers de bronziers ${ }^{12}$. Les fragments de tiges de section circulaire et quadrangulaire sont probablement des déchets ou des ratés de fonte négligés par l'artisan, voire des préformes d'objets.

Le mobilier en alliage cuivreux est complété par un unique morceau de fer de forme triangulaire (US 02). Il mesure $2,6 \times 1,6 \mathrm{~cm}$ et pèse $3,47 \mathrm{~g}$ dans son état actuel. Très corrodé, il est recouvert de concrétions irrégulières. Il pourrait s'agir d'un déchet lié à un travail de forge ou d'un objet cassé, abandonné dans la fosse.

12. Pernot 1998 


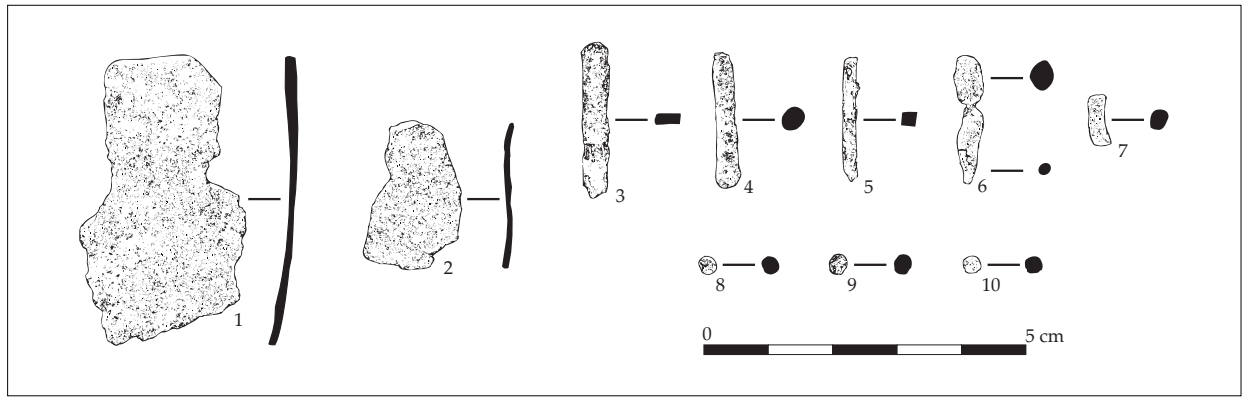

Fig. 5. ZAC de Belle Aire Sud à Aytré, Charente-Maritime. Mobilier métallique (alliage cuivreux) de la fosse 21 (dessin et DAO Chr. Maitay, INRAP).

Le mobilier céramique et métallique était accompagné de quelques pièces lithiques. Il s'agit de petits galets de quartzite et d'un morceau de grès, dont l'une des faces présente des traces de polissage (fragment de meule?). Les vestiges fauniques et malacologiques sont représentés par quelques esquilles osseuses indéterminables et par deux coquilles de patelle.

\section{AtTribution CHRONOLOGIQUE}

\section{Les formes céramiques}

Le mobilier céramique découvert dans la fosse 21 présente de nombreuses affinités avec les séries du premier âge du Fer régional. Certains décors et traits morphologiques s'inscrivent encore dans un contexte de la fin de l'âge du Bronze (jatte à panse convexe et lèvre arrondie : fig. $4 \mathrm{a}, \mathrm{n}^{\circ} 3$; vase de stockage à haut col évasé : fig. $4 \mathrm{a}, \mathrm{n}^{\circ} 5$; cordons et lèvres digités : fig. $4 \mathrm{~b}, \mathrm{n}^{\circ} 6$ à 8 ; larges cannelures : fig. $4 \mathrm{~b}$, $\left.\mathrm{n}^{\circ} 15\right)$, mais les formes carénées à col court subvertical (fig. $4 a, n^{\circ} 1$ et 2 ) sont en revanche bien caractéristiques de la phase moyenne du premier âge du Fer régional ${ }^{13}$.

Le petit vase à épaulement marqué, col subvertical et lèvre arrondie (fig. $4 \mathrm{a}, \mathrm{n}^{\circ} 1$ ) est une forme caractéristique de la fin de la phase ancienne et de la

13. Maitay et al. $2009: 315$. phase moyenne du premier âge du Fer. Il s'agit ici du premier exemplaire connu pourvu d'un décor. Les récipients à panse segmentée se retrouvent sur plusieurs sites charentais de la phase ancienne ou de la phase moyenne du premier âge du Fer : la Combe des Vaux à Echebrune ${ }^{14}$, le Moulin de Reigner à Léoville $^{15}$ et les Planches à Saint-Maigrin ${ }^{16}$, en CharenteMaritime ; Merpins ${ }^{17}$ et les Petits Clairons II à Barbezieux ${ }^{18}$, en Charente. Des détails peuvent varier d'un vase à l'autre (angle du col, forme de l'épaulement), mais le profil général du récipient reste le même. Le fragment de panse carénée ornée d'incisions obliques de Ludène à Loire-les-Marais, dans le nord de la Charente-Maritime ${ }^{19}$, peut être comparé à celui d'Aytré, mais le mobilier qui lui était associé évoque plutôt un contexte de la phase ultime de l'âge du Bronze (jattes à panse ovoïde et col court subvertical ou évasé, vases de stockage à col évasé et lèvre à méplat interne, lignes incisées horizontales sur la partie la plus large de la panse ou sous le col, fines cannelures jointives sur la partie supérieure de la panse, cordons et impressions digités). Les vases du tumulus de Noailles, en Corrèze $\mathrm{e}^{20}$, associés à une épée hallstattienne en bronze de type Miers, fournissent une datation à la phase ancienne du premier

14- Bouchet 1993, fig. 5 et 6 .

15- Marchadier et al. 2005, pl. 21, n4.

16- Burnez \& Réaux 1990, fig. $4, n^{\circ} 23$ et fig. $5, n^{\circ} 4$.

17- Gomez de Soto 1978 , fig. $6, n^{\circ} 9$ et 10

18. Baigl et al. 1999a, fig. 18, n¹8.

19. Favre 1980, pl. 3, n6.

20- Couchard \& Arnal 1963 ; Milcent 2004, 506-507. 
âge du Fer (Hallstatt C1 de la chronologie allemande).

Les vases à épaulement, col concave et lèvre arrondie (fig. $4 \mathrm{a}, \mathrm{n}^{\circ} 2$ ) se retrouvent assez fréquemment sur des sites régionaux du premier âge du Fer. Les structures domestiques fouillées aux Petits Clairons II à Barbezieux, en Charente, en ont livré plusieurs exemplaires, de l'étape moyenne du premier âge du Fer ${ }^{21}$. Des récipients de forme semblable, attribués au Hallstatt C2 et D1, proviennent d'Echebrune $^{22}$, des Planches à Saint-Maigrin ${ }^{23}$ ou de l'enclos 26 de Saint-Georges-de-Didonne ${ }^{24}$, en Charente-Maritime. Les jattes et bols à panse convexe et lèvre aplatie ou arrondie (fig. $4 a, n^{\circ} 3$ ) constituent un marqueur chronologique beaucoup moins fiable. On les rencontre sur des sites d'habitat et dans des grottes de l'âge du Bronze (notamment final : Fort Clan à Cenon-sur-Vienne ${ }^{25}$, le Camp Allaric à Aslonnes ${ }^{26}$, dans la Vienne ; la grotte du Quéroy à Chazelles, en Charente ${ }^{27}$ ) ou de l'âge du Fer (Soubérac à Gensac-la-Pallue $^{28}$, en Charente ; Yves ${ }^{29}$, en Charente-Maritime). Cette forme largement ouverte et pourvue d'un fond plat constitue l'un des récipients les plus fréquents dans l'ouest de la France pendant l'âge du Bronze et le premier âge du Fer.

Les grands vases de stockage à panse ovoïde ou piriforme et haut col évasé (fig. $4 a, n^{\circ} 5$ ) sont également fréquents dans les corpus de la fin de l'âge du Bronze (Fort $\mathrm{Clan}^{30}$, le Camp Allaric, etc.), et du premier âge du Fer (les Petits Clairons II à Barbezieux ${ }^{31}$, etc.). Les cols évasés à lèvre digitée sont davantage spécifiques du premier âge du Fer (fig. 4b, n⿳6) ; ils demeurent encore rares dans les corpus de l'âge du Bronze. Les grands vases de stockage à haut col évasé et lèvre aplatie vers l'intérieur (fig. $4 a, n^{\circ} 5$ ) sont bien attestés dans les corpus du Bronze final ${ }^{32}$ et perdu-

21. Baigl et al. 1999a, fig. $12, n^{\circ} 104$, fig. $17, n^{\circ} 7,14$ et 15 .

22. Bouchet 1993, fig. 5 (repris dans Marchadier et al. 2005,

pl. $11, \mathrm{n}^{\circ} 1$ à 5 ; type $\mathrm{BF} 2$ "jattes segmentées à ouverture convergente").

23. Burnez \& Réaux 1990, fig. 5, n²1.

24- Baigl et al. 1999b, pl. 1, iso 2.

25- Maitay 2001, pl. 3 et 11.

26- Maitay coord. 2009, 28.

27. Gomez de Soto \& Kerouanton 1991, fig. 28, 38...

28- Burnez 1965, fig. 7, n¹.

29. Favre 1998 , pl. 2, n $^{\circ} 6$ et 9.

30- Maitay 2001, pl. 26, n³, pl. 27, pl. 28, n² à 6 .

31- Baigl et al. 1999a.

32. Maitay 2001, pl. $26, n^{\circ} 1$ et 2 . rent longtemps pendant le premier âge du Fer. On en connaît par exemple à Merpins ${ }^{33}$, en Charente, mais aussi à Echebrune ${ }^{34}$, au Crapaud à Fléac-surSeugne $^{35}$, à Ludène à Loire-les-Marais ${ }^{36}$ et à Pérignac $^{37}$, en Charente-Maritime.

\section{Les décors}

Dans le centre-ouest de la France et le Limousin, les décors incisés, cannelés, etc., sont relativement peu fréquents sur les céramiques du premier âge du Fer. Le riche répertoire de motifs géométriques et de signes incisés du Bronze final IIIb, parfois rehaussé d'enductions rouges et/ou noires, n'est plus utilisé sur les céramiques des phases ancienne et moyenne du premier âge du Fer. Les décors plastiques sont très rares et se résument le plus souvent à des impressions et à des cordons digités. Quant aux registres peints, relativement abondants au Bronze final IIIb, ils tombent en désuétude au début du premier âge du Fer et ne reviennent sur le devant de la scène qu'à la phase finale (Hallstatt D2/D3-La Tène A ancienne) $)^{38}$.

Le vase à panse décorée de fines incisions obliques (fig. $4 \mathrm{a}, \mathrm{n}^{\circ} 1$ ) trouve de très rares parallèles dans la région. Il peut notamment être mis en relation avec un fragment de panse carénée de Ludène à Loire-les-Marais, en Charente-Maritime, portant également un décor ${ }^{39}$. D'autres exemplaires de décor incisé sont connus à Saint-Denis-d'Oléron, en Charente-Maritime ${ }^{40}$, mais y sont associés à des éléments de formes et de décors appartenant encore à la tradition de la fin de l'âge du Bronze (assiettes et écuelles à décor interne ou rebord facetté, vases à col cylindrique et col court évasé, etc.). Le récipient en terre cuite à base ornée de fines incisions (fig. 4b, $\mathrm{n}^{\circ} 9$ ) ne connaît pas d'exemplaire similaire, les parties inférieures de vases du premier âge du Fer ne recevant qu'exceptionnellement un décor.

\footnotetext{
33. Marchadier et al. 2005, pl. 26, n6 et pl. 29, n ${ }^{\circ} 42$.

34- Bouchet 1993, fig. 6, n³2, fig. 7, n²1, 33 et 34 .

35- Burnez 1986 (repris dans Marchadier et al. 2005, pl. 16, $\left.\mathrm{n}^{\circ} 13\right)$.

36- Favre 1980, pl. $1, \mathrm{n}^{\circ} 2$ et 3.

37. Bouchet et al. 1992 (repris dans Marchadier et al. 2005, pl. $\left.35, \mathrm{n}^{\circ} 17\right)$

38- Maitay 2007 et 2010 ; Maitay et al. 2009 : 317.

39. Favre 1980, pl. 3, n6.

40- Joussaume et al. 1969, fig. 7, n85.
} 
Plusieurs sites d'Aunis et de Saintonge ont livré des cols évasés ou subverticaux à lèvre digitée ( $\mathrm{Lu}$ dène ${ }^{41}$, Challignac ${ }^{42}$, Courcoury ${ }^{43}$, etc.), mais les contextes de découverte ne permettent pas de préciser leur position chronologique à l'intérieur du premier âge du Fer. Les registres de cannelures larges et peu profondes sont eux aussi un héritage du Bronze final et ne peuvent être employés comme marqueur chronologique. Dans la région, on les connaît notamment sur les gisements du premier âge du Fer de Challignac $^{44}$, en Charente, ou de Loire-les-Marais ${ }^{45}$, en Charente-Maritime.

\section{Conclusions}

Il est difficile de se prononcer sur la fonction initiale de la fosse 21. L'absence de trace de chauffe sur les parois semble exclure l'hypothèse d'une utilisation comme structure de combustion. Il aurait pu s'agir d'une fosse destinée à recueillir de la matière première ou bien à supporter un élément vertical de bois. La faible surface dégagée lors du diagnostic ne permet malheureusement pas de dire si cette fosse est réellement isolée ou bien si elle intègre un système plus dense de structures. Le dépôt de sédiment très fin de couleur brun clair (US 03) indique que la structure est restée à l'air libre quelque temps avant d'être réemployée comme dépotoir. Comme l'atteste l'homogénéité du remplissage, celui-ci semble mis en place de manière assez brève. La présence des scories et des gouttelettes de fonte plaide en faveur d'activités artisanales liées à la fabrication d'objets en alliage cuivreux localisées à proximité de la fosse. Excepté les fragments de tiges qui pourraient peut-être être mis en relation avec la fabrication d'épingles ou de fibules, il n'est pas possible de déterminer la nature des objets qui ont pu être fabriqués.

Le mobilier céramique de Belle Aire forme un ensemble homogène. Malgré l'état de fragmentation important des récipients, quelques profils, même incomplets, constituent de bons marqueurs chronologiques. Les vases à épaulement marqué et col court subvertical ne sont connus que sur des sites d'habitat de la fin de la phase ancienne ou de la phase moyenne du premier âge du Fer (Hallstatt C2 et D1 de la chronologie allemande). Les traits morphologiques et stylistiques les plus significatifs des céramiques de la fin de l'âge du Bronze ne se retrouvent pas ici (absence de forme basse et ouverte à rebord facetté, d'enductions peintes monochromes ou polychromes ou d'ornements complexes réalisés par incision), pas plus que les caractères typiques de la fin du premier âge du Fer (haut pied creux, vases à haut col concave s'achevant par une lèvre arrondie, profils à carène très anguleuse, céramique à décor graphité). Cette série peut donc être mise en parallèle avec celles des habitats des Petits Clairons II à Barbezieux, en Charente, d'Echebrune et des Terres Blanches à la Génétouze ${ }^{46}$, en Charente-Maritime, ou avec l'ensemble récemment dégagé aux Terres Rouges à Parthenay, dans les Deux-Sèvres ${ }^{47}$. Les phases ancienne et moyenne du premier âge du Fer manquent encore aujourd'hui cruellement de documentation dans la région ${ }^{48}$, et le mobilier d'Aytré, bien que modeste, apporte un supplément d'information des plus utiles.

41- Favre 1980, pl. 3, nº

42. Burnez et al. 1997, fig. $31, n^{\circ} 9$ et 21 ; Burnez et al. 1998 , fig. $37, n^{\circ} 9$ et 10 , fig. $39, n^{\circ} 6$ et 7 .

43. Marchadier et al. 2005, pl. 10, $n^{\circ} 1$.

44- Burnez et al. 1997, fig. 31, n¹3 ; Burnez et al. 1998, fig. 38, $\mathrm{n}^{\circ} 1$ à 3.

45. Favre 1980, pl. 2, nº 10.

46- Maurel \& Marchadier 2008

47. Bolle et al. 2009.

48- Maitay et al. 2009. 


\section{Bibliographie}

Baigl, J.-Ph., en collab. avec J. Gomez de Soto, Ph. Poirier, I. Kerouanton et E. Bayen (1999a) : "Barbezieux, Les Petits Clairons (Charente). Un établissement rural du premier âge du Fer", Aquitania, 16, 31-91

Baigl, J.-Ph., en collab. avec B. Boisseau, C. Ballarin, L. Bernard, P. Fouéré et FI. Verdin (1999b) : Sanctuaire protohistorique, structures rurales et ensembles funéraires du Haut-MoyenAge, ferme post-médiévale. Déviation de Royan - Phase 1. Saint-Georges-de-Didonne (Charente-Maritime), DFS, SRA de Poitou-Charentes, AFAN, Poitiers.

Bertrand I., A. Duval, J. Gomez de Soto et P. Maguer, dir. (2009) : Actes du XXXle colloque de l'AFEAF, Chauvigny, 17-20 mai 2007, Association des publications chauvinoises-AFEAF, Chauvigny.

Bolle, A., M. Cavaillès, A.-M. Cottenceau-Boullé, J. Gomez de Soto et M. Pichon dir. (2009) : Et avant Parthenay ? Le site prégaulois des Terres Rouges, catalogue de l'exposition présentée au musée municipal de Parthenay, Parthenay.

Bouchet, J.-M. (1993) : La Combe des Vaux, Echebrune (CharenteMaritime), rapport de sauvetage, SRA de Poitou-Charentes, Poitiers

Bouchet, J.-M., C. Burnez et P. Fouéré (1992) : Prospections sur la commune de Pérignac (Charente-Maritime), rapport de prospection, SRA de Poitou-Charentes, Poitiers.

Burnez, Cl. (1965) : "La station de Soubérac à Gensac-la-Pallue (Charente)", Bulletin de la Société préhistorique française, 62, 289-315.

- (1986) : Fléac-sur-Seugne. Le Crapaud, rapport de sauvetage, SRA de Poitou-Charentes, Poitiers.

Burnez, Cl. et B. Réaux (1990) : Les Planches à Saint-Maigrin (Charente-Maritime), rapport de sauvetage, SRA de PoitouCharentes, Poitiers.

Burnez, Cl., C. Louboutin et F. Fischer, dir. (1997) : Le Camp, à Challignac (Charente), rapport de fouille programmée, SRA de Poitou-Charentes, Poitiers.

- (1998) : Le Camp, à Challignac (Charente), rapport de fouille programmée, SRA de Poitou-Charentes, Poitiers.

Couchard, J.-L. et J. Arnal (1963) : "Le tumulus de la Route-Vieille à Noailles, près de Brive (Corrèze)", Gallia-Préhistoire, 6, 133147

Favre, M. (1980) : "Habitat protohistorique de Ludène, commune de Loire-les-Marais (Chte-Mme)", Roccafortis, 4, 118-124.

- (1998) : "Une fosse du premier âge du Fer au Bas des Terres, commune d'Yves, Charente-Maritime", Roccafortis, 3, 225-227.

Gomez de Soto, J. (1978) : Merpins avant le Moyen Âge. L'oppidum de Merpins du Néolithique au IN S. ap. J.-C., Rapport d'activités 1975-1976, Groupe Archéologique Marpenois.

Gomez de Soto, J. et I. Kerouanton (1991) : "La grotte du Quéroy à Chazelles (Charente). Le Bronze final IIlb", Bulletin de la Société préhistorique française, 88 (1997), 341-392.

Hantzpergue, P. (1988) : Carte géologique de la France (1/50000), feuille La Rochelle - Ile de Ré (632-633), éditions du BRGM, Orléans.

Joussaume R., J.-P. Mohen et P. Tardy (1969) : "Préhistoire à Saint-
Denis d'Oléron (Charente-Maritime)", Bulletin de la Société préhistorique française, 56, 457-469.

Maguer P., G. Landreau, C. Dupont, H. Martin, X. Bardot, G. Pouponnot, D. Briand et A. Duval (2009) : "L'habitat littoral des Ormeaux à Angoulins (Charente-Maritime). Activités vivrières et salicoles entre marais et océan", in : Bertrand et al. 2009, 57-102.

Maitay, Chr. (2001) : Une occupation de l'âge du Bronze final IIlb dans la basse vallée du Clain : le site de Fort Clan, à Cenon (Vienne), Mémoire de Maîtrise inédit, UFR Sciences Humaines et Arts, Université de Poitiers.

- (2007) : Les céramiques peintes non tournées dans le CentreOuest de la France et ses marges, de l'âge du Bronze à La Tène A ancienne. Apport à la connaissance des cultures matérielles de l'ouest de la France, Thèse de doctorat, Université de Rennes 1.

- (2010) : Les céramiques peintes pré-celtiques de l'Ouest de la France, Rennes.

- (en préparation): "L'occupation rurale du premier âge du Fer des Drouillards à Dompierre-sur-Mer, Charente-Maritime", Aquitania.

- coord. (2009) : Feux de Camp. Un site de hauteur : le Camp Allaric, du Néolithique à l'âge du Fer, catalogue de l'exposition présentée au Musée Sainte-Croix, Musée de la ville de Poitiers et de la Société des antiquaires de l'Ouest, Poitiers.

Maitay, Chr. et E. Marchadier, en collab. avec B. Béhague (2009) : "Entre traditions locales et apports exogènes : évolutions et singularités de la céramique du premier âge du Fer et de La Tène $A$ ancienne entre Loire et Dordogne", in : Bertrand et al. 2009, 307-340.

Marchadier, E., en collab. avec J.-M. Bouchet, Cl. Burnez et J. Gomez de Soto (2005) : Premier âge du Fer en Saintonge et Aunis : étude typo-chronologique du mobilier céramique, Société d'archéologie et d'histoire de la Charente-Maritime (2007), Saintes.

Maurel, L. et E. Marchadier (2008) : "Le site 'Les Terres Blanches' à La Génétouze : un épandage de mobilier céramique daté du premier âge du Fer", Bulletin de l'Association des archéologues de Poitou-Charentes, 38, 39-46.

Maurin, L. (1999) : La Charente-Maritime, CAG 17, Paris.

Milcent, P.-Y. (2004) : Le premier âge du Fer en France centrale, Société préhistorique française, Mémoire XXXIV, Paris.

Nibodeau, J.-P. (2008) : Aytré (Charente-Maritime). ZAC de Belle Aire Sud, rapport de diagnostic d'archéologie préventive, SRA de Poitou-Charentes, INRAP, Poitiers

Pautreau, J.-P. (1976) : "Le Camp Allaric, commune d'Aslonnes (Vienne). Premiers résultats", L'Anthropologie, 80, 389-429.

Pernot, M. (1998) : "L'organisation de l'atelier de bronzier", in : Mordant C., M. Pernot et V. Rychner éd., L'atelier du bronzier en Europe $d u x x^{e}$ au vIII siècle avant notre ère. t. II. Du minerai au métal, du métal à l'objet, Paris, 107-116.

Soler, L., en collab. avec M.-C. Arqué, L. Bedault, C. Gay, J. Mousset et E. Normand (2008) : Le Fief des Quatre Chevaliers à Périgny (Charente-Maritime). Zone industrielle de La Rochelle, RFO de fouille archéologique, SRA de Poitou-Charentes, Conseil général de la Charente-Maritime.

Vacher, S., dir. (en préparation) : Le site de l'âge du Bronze ancien de la Vaurie à Périgny, Charente-Maritime, RFO de fouille archéologique, SRA de Poitou-Charentes, INRAP, Poitiers. 
